\title{
Different Methods of Probiotics Stabilization
}

\author{
Kamila Goderska
}

Additional information is available at the end of the chapter

http://dx.doi.org/10.5772/50313

\section{Introduction}

Starter cultures provide a basis in the production of fermented foods. Probiotics are the most important group of bacterial starter cultures. Commercial starter cultures were initially supplied in liquid form prior to the production of concentrated starter cultures. Progress in biotechnology later led to the application of concentrated starter cultures in frozen and freeze dried forms for direct incorporation into the food formulation. Application of frozen or freeze-dried starter cultures eliminates in -plant sub-culturing, reduces the costs associated with bulk culture preparation and lowers the risk of bacteriophage infection (Desmod et al. 2002).

Very low transportation and storage temperatures are the main commercial disadvantages of frozen starter cultures (Ghandi et al. 2012). Besides the risk of thawing, high transportation costs may limit the use of frozen starter cultures in distant areas or countries. Starters of probiotic bacteria are usually preserved by freeze thawing and lyophilization. In spite of being efficient methods, freezing and freeze drying have high manufacturing costs and energy consumption. For this reason, increasing attention has been paid on alternative dring processes such as spray drying, fluidized bed drying and vacuum drying.

Majority of vegetative forms of microorganisms are characterized by poor thermostability. They exhibit considerably high rates of dying and loss of activity as a result of thermal inactivation at the range of temperatures from 40 to $60^{\circ} \mathrm{C}$. With regard to microbial biomass, there is certain critical water content (depending on the object property) which, when exceeded, results in dehydration inactivation. This can be attributed to the fact that in the case of vegetative forms of microorganisms water does not only provide environment for their life but it also acts as a substrate for biochemical reactions and its removal below a certain level prevents maintenance of metabolic functions and, consequently, leads to the death of cells. Among dehydration methods which allow maintaining viability of microbial 
biomass are: freeze-drying, sublimation drying, including fluidization drying using inert materials (carriers) and spray drying (Santivarangkna et al. 2008).

Freeze drying is therefore more convenient and easier as it does not require freezing conditions during distribution. Although freeze drying is the conventional drying technique used commercially by starter culture manufactures, it is lengthy and more expensive than other drying processes (Fonseca et al 2001, Ampatzoglou et al. 2010, Morgan et al. 2006). Many attempts have been made to develop alternative drying processes at lower cost and some authors have reported reasonable cell viability after drying (Tymczyszyn et al. 2008).

Spray drying is considered a good long-term preservation method for probiotic cultures. The spray drying of microorganisms dates to 1914 to the study of Rogers on dried lactic acid cultures. The concept of spray drying was first patented by Samuel Percy in 1872, and its industrial application in milk and detergent production began in the 1920s. The speed of drying and continuous production capability are very useful for drying large amounts of starter cultures. Since then, much research has been reported on the spray drying of bacteria without loss of cell activity in order to overcome the difficulties involved in handling and maintaining liquid stock cultures.

Spray drying is a unique process in which particles are formed at the same time as they are dried. It is a very suitable for the continuous production of dry solids in powder, granulate or agglomerate form liquid feed stocks as solutions, emulsions and pumpable suspensions. The end product of spray drying must comply with precise quality standards regarding particle size distribution, residual moisture, bulk density, and particle shape. In the spray drying process, dry granulated powders are produced from a slurry solution, by atomizing the wet product at high velocity and directing the spray of droplets into a flow of hot air e.g. $150-200^{\circ} \mathrm{C}$. The atomized droplets have a very large surface area in the form of millions of micrometer-sized droplets $(10-200 \mu \mathrm{m})$, which results in a very short drying time when exposed to hot air in a drying chamber (Sunny-Roberts, Knorr 2009).

Spray drying involves atomization of a liquid feedstock into a spray of droplets and contacting the droplets with hot air in a drying chamber. The sprays are produced by rotary (wheel) or nozzle atomizers. Evaporation of moisture from the droplets and formation of dry particles proceed under controlled temperature and airflow conditions. Powder is discharged continuously from the drying chamber (Peighambardoust et al. 2011).

Spray drying is a common industrial and economic process for the preservation of microorganisms and for the preparation of starter cultures that are used to prepare lacticfermented products. The survival of lactic acid bacteria is an important issue when spray drying is used for the preparation of microbial cultures. However biological activity of a lactic acid starter, which includes cell viability and physiological state, is a criterion for evaluating starter quality (Carvalho et al. 2004, Ananta et al. 2005).

It has been shown that both the water evaporation rate and the temperature of droplets containing microbial cells have a significant effect on their survival during spray drying. 
Since it is not yet possible to quantify the changes occurring in the bacterial cells and their survival in situ when they are subjected to spray drying, single droplet drying is used instead. Single droplet drying, in which a single droplet is suspended in moving and conditioned air, provides the closest experimental resemblance to the spray drying environment. Single droplet drying can be conducted in various ways, for example (a) a single or a stream or streams of droplets could be allowed to fall under gravity in a towerlike dryer, (b) a droplet can be freely levitated using ultrasonic or aerodynamic fields, or (c) a droplet can be suspended on the tip of a fine glass filament. The first two method are not very popular as they are expensive and the heat and mass transfer rates in these environments are not close to the convective drying environment of spray drying. Li et al (2006) investigated the inactivation kinetics of two probiotic strains (Bifidobacterium infantis and $S$. thermophilus) in air temperature and relative humidity in the ranges of $70-100{ }^{\circ} \mathrm{C}$ and $3,7-0,5 \%$, respectively, using single droplet drying in skim milk as a suspending medium. They reported that the inactivation mainly occurred at the early stage of the drying when the evaporation rate was high. The above studies do not offer a unanimous view whether the drying rate or the droplet temperature is the limiting factor of bacterial survival during drying.

Freeze drying is a preferred drying method for thermally sensitive bacteria as it keeps their survival at a reasonably high level. However, freeze drying is a batch process with a considerably long drying time. It is also expensive due to high energy requirements. For drying of starter cultures, spray drying can be a viable alternative if the survival can be raised to make it economically attractive. This is because spray drying is relatively inexpensive, energy efficient, high throughput and a hygienic process (Papapostolou et al. 2008, Carvalho et al. 2004).

In order to minimise cell death, the effects of drying parameters (inlet and outlet air temperatures, air flow rate, relative humidity, residence time, protective agents) on the survival and vitality of bacteria have to be understood to a considerable depth. The drying process causes damage to the cell wall and cellular components, especially cytoplasmic membrane and proteins, which results in the loss of survival. This cellular injury leads to cell inactivation and negatively impacts the productivity and characteristics of dried culture, and hence the cellular injury has to be minimised. Protective agents such as carbohydrates, proteins, amino acids, gums and skim milk are used to minimise the bacterial inactivation during drying. It is reported that low molecular weight carbohydrates such as sugars stabilize the membrane and protein chains of cellular macromolecules in dry state through hydrogen bonding in lieu of water when the water molecules are removed through desiccation. Protein are capable of forming relatively stable intracellular glasses, and by doing so, they can be more effective a protective materials for bacterial culture than sugars. It is reported that the combination of different protectans (e.g. mixtures of sugar and protein) can have synergistic effect on cell viability rather than acting individually. It has been shown that both the water evaporation rate and the temperature of droplets containing microbial cells have a significant effect on their survival during spray drying. 
Encapsulation of probiotics is employed in order to increase the bacteria resistance to freezing and freezing drying of the food. In most of the studies the probiotic bacteria were entrapped in a gel matrix of biological nature materials such as alginate, $\kappa$-carrageenan, and gellan/xanthan (Semyonov et al. 2010, Kanmani et al. 2011). The core and wall solution was turned into drops of desired size by an extrusion method, employing an emulsion or by transfer from organic solvents. One problem in the probiotic entrapment approach is that the gel beads technologies stabilize the bacteria mostly in liquid products, and are difficult to scale up. To extend storage shelf-life $t$ is convenient to convert the micro-capsules into a dry powder by employing techniques such as spray drying, freeze drying, and/or fluidized bed drying. The spray drying is an economic and effective technology, however, it causes high mortality as a results of simultaneous dehydratation, thermal and oxygen stresses imposed to bacteria during the drying process. Freeze drying is considered one of the most adequate methods for drying biological materials and sensitive foods. However, when this method was employed for drying probiotic bacteria and other cells, undesirable effects such leakage of the cell membrane due to changes in the physical state of membrane lipids or changes in he structure of sensitive proteins in the bacteria cell occur. Protective solutes such as cryoprotectans (saccharides and polyols) and other compatible solutes like adonitol, betaine, glycerol and skim milk were used to increase bacteria's viability and increase their survival during freeze-drying and subsequent storage. These studies lead to the conclusion that the effect of each protective agent on the viability of a specific lactic acid bacteria strain during or following the freeze-drying process have to be determined on a case-by-case basis (Heidebach et al. 2010, Krasaekoopt et al. 2003).

As mentioned above, dried probotic micro-capsules can be coated by an additional layer (shell) in order to protect the bacterial core from the acidic environment of the stomach and to avoid the deleterious effect of bile salts on the cell's membrane. This additional shell can help to release the bacterial core at a desired site in the GIT. In order to be further coated, bulk freezed powders are micronized to a narrow particle distribution. This process is complex, requires intensive energy, and decrease the viability of the dried cells.

The pharmaceutical industry utilized recently the spray freeze drying for pharmaceutical powders preparation. This method combines the narrow article size distribution of an extrusion device and the freeze-drying process to prepare a dry powder of desired particle size and of the narrow distribution. Spray freeze drying basic principle is to spray a solution containing dissolved/suspended material (e.g. protein) by an atomization nozzle into a cold vapor phase of a cryogenic liquid, such a liquid nitrogen, so the droplets may start freezing during their passage through the cold vapor phase, and completely freeze upon contact with the cryogenic liquid phase. The frozen droplets are then dried by lyophilization (Lian et al. 2002, Gardiner et al. 2002).

Spray freeze drying powders have a controlled size, larger specific surface area and a better porous character than spray-dried powders. The particles retain their spherical and porous morphology and can be further coated with an enteric food grade biological polymer which is designed to desintegrate at specific loci in the GIT. 
Recently this method was further developed and the solution is sprayed under adequate pressure via a needle directly in liquid nitrogen. The cooling rates in the spray freezing section are dependent on many factors and thus are also very difficult to estimate. However it was claimed that maximum cooling rates by freezing in liquid nitrogen are the order of $300 \mathrm{~K} / \mathrm{s}$, considered as upper boundary for the cooling rate. To the best of our knowledge the spray freeze drying method was not used yet to produce dry powder of probiotic cells.

Vacuum drying has been described to be the most promissory method to reserve sensible biological material because of its acceptable cost-effectiveness balance. However, the conditions of vacuum drying (time, temperature) must be optimized to allow the best bacterial recovery after dehydratation-rehydratation, avoiding cellular damages (Tymczyszyn et al. 2008).

It has been proposed that bacterial death results from the inactivation of critical sites in the cells. Membranes, nucleic acids and certain enzymes have been identified as cellular targets of damage caused by dehydratation. It has been reported that after dehydratationrehydratation the microorganisms can be recovered even when the cellular membrane is damaged. In addition, it has also been observed that an increase in the absolute value of the zeta potential can be associated with an increase in the lag time. Changes in this parameter were correlated with a loss of the original orientation of the surface macromolecules and thus, the capacity to recover the surface properties after rehydratation. This indicates that there are other bacterial structural parameters besides the membrane integrity affecting the bacterial viability after dehydratation-rehydratation. In this sense, date obtained by Differential Scanning Calorimetry reveal that damage produced in membrane lipids, ribosomes and DNA are reversible, whereas damages produced in proteins are not.

When applying vacuum drying, it is important to consider that a thermal stress takes place in parallel to the hydric stress, probably inducing irreversible damages. For this reason, the exposure of microorganisms to high temperatures should be as short as possible and the correct choice of times and temperatures of dehydratation is crucial to achieve the best vacuum drying conditions.

The challenge of making vacuum drying a wide spread methodology for microorganisms' preservation is the difficulty of defining standardized conditions that allow the comparison of results obtained in different laboratories. The reason of the difficulty is that the times and temperatures for the dehydratation processes are related with the drying conditions (i.e.: exposure surface, pressure of the vacuum system, weight or volume of the sample, etc.), which in general are dependent on the equipment used. Therefore, to make results comparable, it becomes necessary to refer the experimental conditions, to a parameter that is independent to these experimental conditions, for example, the water activity of the sample after dehydratation in a given condition.

I consequence, considering that both time and temperatures of drying affect the final water activities of the samples, the definition of drying conditions in terms of the final water activity becomes important to define correlatable parameters with the state of dehydratation of the cells. This fact would help to attain the best conditions for the preservation processes. 
Bifidobacteria benefit human health by improving the balance of intestinal microbiota and by strengthening mucosal defenses against pathogens. However, for probiotics to be therapeutically effective, it has been suggested that products should contain at least $6 \log$ $\mathrm{cfu} / \mathrm{g}$ of bacteria until the end of their shelf life. Although bifidobacteria are being increasingly recognized as probiotics that have advantageous properties, they are also fastidious, obligate anaerobes and, therefore, pose a technological challenge for the food industry. several factors have been claimed to affect the viability of bifidobacteria, including acidity, $\mathrm{pH}$, time and temperature of storage, and oxygen content.

Within this context, microencapsulation of probiotic bacteria is currently drawing more and more attention for being a method to improve the stability of probiotic organisms in functional food products. Microencapsulation may improve the survival of these microorganisms, during both processing and storage, and also during passage through the human gastrointestinal tract. Spray drying is regarded as a microencapsulation method and it has been investigated as a means of stabilizing probiotic bacteria in a number of food matrices, most often composed of proteins, polysaccharides, sugars, and combination thereof. The survival rate of the culture during spray drying and subsequent storage depends upon a number of factors, which may include the species and strain of the culture, the drying conditions and also the use of encapsulating agents.

Reconstituted skim milk is an encapsulating agent that has shown a favorable effect on the improvement of cell survival during the spray drying process. Another approach to increase the viability of bifidobacteria is the use of prebiotics, which are nondigestible food ingredients that beneficially affect the host by selectively stimulating the growth and/or activity of bacteria in the colon. Inulin is a prebiotic whose degree of polymerization (DP) ranges between 10 to 60 . It is extracted from chicory roots and consists of chains of fructose units. Oligofructose is obtained throught partial hydrolysis of inulin and therefore has a lower DP,, which range from 2 to 8 . A mixture of oligofructose and inulin is known as oligofructose-enriched inulin. These prebiotics may potentially be exploited as carrier media for spray drying and may be useful for enhancing probiotic survival during processing. However, the use of different encapsulatin agents for production of microcapsules can result in different physical properties, depending on the structure and the characteristics of each agent. (Fritzen-Freire et al. 2012).

The study was conducted to evaluate the viability and the physical properties of Bifidobacterium BB-12 microencapsulated by spray drying partial replacement of reconstitutet skum milk (RSM), as encapsulating agent with the prebiotics inulin, oligofructose, and oligofructose-enriched inulin (at ratio of 1:1, 200g/ total concentration). The viable cell counts of the microcapsules were determined during storage for 180 days at $4^{\circ} \mathrm{C}$ and at $-18^{\circ} \mathrm{C}$. The partial replacement of RSM with inulin and the partial replacement of RSM with oligofructose-enriched inulin increased the initial count of bifidobacteria in the microcapsules. On the other hand, the microcapsules produced with oligofructose-enriched inulin and those produced with oligofructose showed better protection for the bifidobacterium during storage. The use of prebiotics did not affect the morphology of the 
microcapsules. However, the capsules produced with oligofructose showed a smaller particle size. The inclusion of prebiotics decreased the moisture content and water activity in the microcapsules. The microcapsules produced with inulin showed the lowest dissolution in water, while the microcapsules produced with oligofructose were the most hygroscopic. The total color difference of the microcapsules was not considered obvious to the human eye. The results of the thermoanalyses suggest an increase in the stability of the microcapsules produced with prebiotics. Finally, the results showed that the oligofructoseenriched inulin is the most appropriate prebiotic to be used as partial replacement of RSM to microcapsulate Bifidobacterium BB-12 by spray drying, with a great potentail as a functional ingredient to be applied in dairy foods. (Fritzen-Freire et al., 2012).

Ultrasonic vacuum spray dryer was used to produce a dry powder of highly viable probiotic cell. The drying was performed through two stages: vacuum spray drying of the solution followed by fluidized-bed drying of the powder. The embedding matrix was a combination of trehalose and maltodextrin. The effect of external and internal variables on cell survival during the drying process and storage were investigated. The hypothesis was that by minimizing the oxidative and thermal stresses in the drying stages, in addition to adequate formulation choice, the cell viability during the drying and storage will increase. It was concluded that during the drying process the faster the embedding matrix reaches a glassy state the higher was the probiotic survival. Evaluating water activity and moisture limit of the glassy matrix concluded that maltodextrin DE5 is a better encapsulating matrix than maltodextrin DE19. Combining trehalose to maltodextrin in the encapsulating matrix resulted in a significant increase in the survival up to $70.6 \pm 6.2 \%$.

Higher temperatures used during spray drying may be detrimental to bacteria. However this is not the case for certain lactic acid bacteria. For example, similar survival rates were obtained on freeze-drying and spray-drying of concentrated cultures of Lactobacillus bulgaricus. cellular damage to probiotics may be reduced and viability preserved through control of drying parameters; specifically, by lowering the outlet temperature of spray dryers and the incorporation of appropriate carriers into the drying medium. The addition of sugars to the growth medium also influences the survival of dried probiotic preparations. The incorporation of glucose in formulations did not markedly influence the survival of probiotic during drying but had marked effects on Lactobacillus GG survival during subsequent long term storage. These results corroborate those of others who also found that although the survival of LGG during spray drying was not significantly affected when different media (reconstituted skim milk (RSM), RSM/polydextrose, RSM-Raftilose P95) were used, it did influenced survival of bacteria during long term storage. A similar result was reported, where the presence of sugars (fructose, trehalose or sucrose) or sugar alcohols (inositol, sorbitol) improved survival of Lactobacillus plantarum and L. rhamnosus during storage but not during the freeze-drying (Yang Ying et al. 2012).

The glucose-containing formulations in the study, improved storage stability of spray-dried LGG microcapsules stored under similar environmental conditions although the glass transition temperature of these formulations was depressed in comparison to those of 
formulations without glucose. It has been suggested that the incorporation of small sugars improves survival of bacteria during drying because of their ability to replace water that is removed from proteins/enzymes within the cells and reduce the membrane phase transition temperature. Results suggest that the effect of glucose is more significant during storage than during drying, even though glucose containing formulations did not maintain its glassy state at different storage conditions. The results of the work are in line with those of others which show that a glassy state during storage alone is not sufficient for stabilization of dried bacterial preparations.

Protectants which preserve the structural integrity of cell membranes, proteins and enzyme functions are required for improving viability during storage of dried probiotic preparations. These results suggest that a pre-requisite for LGG survival in the glassy state is the direct interactions between a low molecular weight sugar and cell components, which helps preserve cell functions during drying with subsequent beneficial effects on long term storage. Both the maintenance of a glassy state during storage and the incorporation of glucose or a low molecular weight sugar in the drying medium are required for optimal survival of probiotic powders during storage (Yang Ying et al. 2012).

The process for the formation of dry-encapsulated probiotics, using ultrasonic vacuum spray drying (UVSD), and microcapsule matrix composed of maltodextrin and trehalose were studied. The results of this study demonstrate thet using UVSD brought the matrix repidly to a glassy state and provided high survival of the probiotic cells- $3.3 \times 10^{9} \mathrm{cfu} / \mathrm{g} \mathrm{dm}$, that was achieved with maltodextrin DE-trehalose (1:1) $20 \% \mathrm{~g} / 100 \mathrm{~g}$ matrix and $7.0 \times 10^{9} \mathrm{cfu} / \mathrm{g}$ $\mathrm{dm}$ initial L. paracasei concentration. It was found that MD DE5 was a better encapsulation matrix than MD DE19, probably due to the fact that DE5 matrix maintained its glassy state at a higher $a_{w}$. The addition of trehalose increased the viability significantly during the drying and during storage of the dried powder. MD DE5-trehalose combination (1:1) resulted with the highest survival $(70.6 \pm 6.2 \%)$. Evidently, further protection should be provided to the cells against oxidation, as storage in nitrogen was essential in order to gain storage stability. (Semyonov et al. 2011)

Improved production methods of starter cultures, which constitute the most important element of probiotic preparations, were investigated. The aim of the presented research was to analyse changes in the viability of Lactobacillus. acidophilus and Bifidobacterium bifidum after stabilization (spray drying, liophilization, fluidization drying) and storage in refrigerated conditions for 4 months. The highest numbers of live cells, up to the fourth month of storage in refrigerated conditions, of the order of $10^{7} \mathrm{cfu} / \mathrm{g}$ preparation were recorded for the B. bifidum DSM 20239 bacteria in which the N-Tack starch for spray drying was applied. Fluidization drying of encapsulated bacteria allowed obtaining a preparation of the comparable number of live bacterial cells up to the fourth month of storage with those encapsulated bacteria, which were subjected to freeze-drying but the former process was much shorter. The highest survivability of the encapsulated Lb. acidophilus DSM 20079 and B. bifidum DSM 20239 cells subjected to freeze-drying was obtained using skimmed milk as the cryoprotective substance. Stabilisation of bacteria by microencapsulation can give a 
product easy to store and apply to produce dried food composition (Goderska, Czarnecki 2008) .

\section{Author details}

Kamila Goderska

Faculty of Food Science and Nutrition, Institute of Food Technology of Plant Origin, Department of Fermentation and Biosynthesis, University of Life Sciences in Poznan, Poland

\section{References}

Ampatzoglou A., Schurr B., Deepika G., Baipong S., Charalampopoulos D. Influence of fermentation on the acid tolerance and freeze drying survival of Lactobacillus rhamnosus GG. Biochemical Engineering Journal 2010, 52, 65-70

Ananta E., Volkert M., Knorr D. Cellular injuries and storage stability of spray-dried Lactobacillus rhamnosus GG. International dairy Journal 2005, 15, 399-409

Carvalho A.S., Silva J., Ho P., Teixeira P., Malcata F.X., Gibbs P. Relevant factors for the preparation of freeze-dried lactic acid bacteria. International Dairy Journal 2004, 14, 835847

Desmond C., Stanton C., Fitzgerald G.F., Collins K., Ross R.P. Environmental adaptation of probiotic lactobacilli towards improvement of performance during spray drying. International Dairy Journal 2002, 12, 183-190

Fonseca F., Beal C., Corrieu G. Operating conditions that affect the resistance of lactic acid bacteria to freezing and frozen storage. Cryobiology, 2001, 43, 189-198

Fritzen-Freire C.B., Prudencio E.S., Amboni R.D.M.C., Pinto S.S., Negrao-Murakami A.N., Murakami F.S. Microencapsulation of bifidoabcteria by spray drying in the presence of prebiotics. Food Research International 2012, 45, 306-321

Gardiner G.E., Bouchier P., O'Sullivan E., Kelly J., Collins J.K., Fitzgerald G., Ross R.P., Stanton C. A spray-dried culture for probiotic Cheddar cheese manufacture. International Dairy Journal 2002, 12, 749-756

Ghandi A., Powell I., Chen X.D., Adhikari B. Drying kinetics and survival studies of dairy fermentation bacteria in convective air drying environment using single droplet drying. Journal of Food Engineering 2012, 110, 4,05-417

Goderska, Czarnecki Influence of microencapsulation and spray drying on the viability of Lactobacillus and Bifidobacterium strains. Polish Journal of Microbiology 57(2), 135-140

Kanmani P., KUmar R.S., Yuvaraj N., Paari K.A., Pattukumar V., Arul V. Effect of cryorpeservation and microencapsulation of lactic acid bacterium Enterococcus faecium MC13 for long-term storage. Biochemical Engineering Journal 2011, 58-59, 140-147

Krasaekoopt W., Bhandari B., Deeth H. Evaluation of encapsulation techniques of probiotics for yoghurt. International Dairy Journal 2003, 13, 3-13

Lian W.C., Hsiao H.C., Chou C.C. Survival of bifidobacteria after spray-drying. International Journal of Food Microbiology 2002, 74, 79-86 
Morgan C.A., Herman N., White P.A., Vesey G. Preservation of micro-organisms by drying; A review. Journal of Microbiological Methods 2006, 66, 183-193

Papapostolou H., Bosnea L.A., Koutinas A.A., Kanellaki M. fermentation efficiency of thermally dried kefir. Bioresource technology 2008, 99, 6949-6956

Peighambardoust S.H., Tafti A.G., Hesari J. Application of spray dring for preservation of lactic acid starter cultures: a review. Trends in Food Sciences \& Technology 2011, 22, 215-224

Santivarangkna C., Higl B., Foerst P. Protection mechanisms of sugars during different stages of preparation process of dried lactic acid starter cultures. Food Microbiology, 2008, 25, 429-441

Semyonov D., Ramon O., kaplun Z., Levin-Brener L., Gurevich N., Shimoni E. Microencapsulation of Lactobacillus paracasei by spray freeze drying. Food Research International 2010, 43, 193-202

Semyonov D., Ramon O., Shimoni E. Using ultrasonic vacuum spray dryer to porduce highly viable dry probiotics. LWT-Food Science and Technology 2011, 44, 1844-1852

Sunny-Roberts E.O., Knorr D. The protective effect of monosodium glutamate on survival of Lactobacillus rhamnosus GG and Lactobacillus rhamnosus E-97800 (E8000) strains during spray-drying and storage in trehalose-containing powders. International dairy Journal 2009, 19, 209-214

Tymczyszyn E.E., Diaz R., Pataro A., Sandonato N., Gomez-Zavaglia A., Disalvo E.A. Critical water activity for the preservation of Lactobacillus bulgaricus by vacuum drying. International Journal of Food Microbiology 2008, 128, 342-347

Ying D.Y., Sun J., Sanguansri L., Weerakkody R., Augustin M.A., Enhanced survival of spray-dried microencapsulated Lactobacillus rhamnosus GG in the presence of glucose. Journal of Food Engineering, 2012, 109, 597-602

Zbicinski I., Delag A., Strumillo C., Adamiec J. Advanced experimental analysis of drying kinetics in spray drying. Chemical Engineering Journal 2002, 86, 207-216 